\title{
Polylactic acid/polyurethane blend reinforced with cellulose nanocrystals with semi-interpenetrating polymer network (S-IPN) structure
}

\author{
Jatin Sethi ${ }^{1,2}$, Mirja Illikainen ${ }^{1}$, Mohini Sain ${ }^{2,3}$, and Kristiina Oksman 1,2,3*
}

\begin{abstract}
The aim of the current work was to prepare and characterize a cellulose nanocrystal reinforced semi-interpenetrated network (SIPN) derived from polylactic acid (PLA) and polyurethane (PU) polymers. SIPN films were prepared using solvent casting from 1,4-dioxane solution. The morphology, mechanical and thermal properties of the neat SIPN and its nanocomposite were characterized. A novel dispersion method was used, for the first time, to disperse the CNCs into the polyol. This method led to well dispersed CNCs in the SIPN, and at $1 \mathrm{wt} \% \mathrm{CNC}$ concentration, the elastic modulus of the nanocomposite was improved by $54 \%$ over an unreinforced SIPN. Additionally, the results indicated that the toughness of PLA, which is the main polymer phase, was improved. However, in the nanocomposite, $\mathrm{CNCs}$ formed a strong network and reinforced the PU phase, which resulted in a lower toughness of the final material. The storage modulus of the SIPN nanocomposite was higher than that of the neat PLA at temperatures higher than $55^{\circ} \mathrm{C}$ up to $100{ }^{\circ} \mathrm{C}$. This increase in thermomechanical properties indicates that the reinforced PU network in the PLA matrix can enhance the thermal behavior of material.
\end{abstract}




\section{Keywords}

Polylactic acid; polyurethane; nanocellulose; semi-interpenetrating networks; dispersion; nanocomposites.

\section{Introduction}

Polymeric composites are currently at a decisive crossroad where researchers are slowly and steadily shifting their focus to a more sustainable bio-based economy; therefore, the field of biomaterials and biocomposites has gained unprecedented attention in the last few years. Apart from being eco-friendly, biocomposites also have multiple additional benefits. They provide a skeleton structure for lightweight materials, which will cater to the needs of the transport industry by reducing the cumulative weight and will hence decrease fuel consumption and greenhouse gases. Although encouraging developments have been made in this field, further research is needed to bring them to a par with petroleum-based composites [1-4].

Bio-based composites lag behind petroleum-based ones in a critical aspect, mechanical properties $[1,5,6]$, which need to be improved for the commercial streamlining of such materials. Currently, the most studied way to improve the properties of biopolymers is by reinforcing them with nanocelluloses (NCs) [7]. NCs, which is a generic term for cellulose nanocrystals (CNCs) and cellulose nanofibers (CNFs), have gained considerable attention due to their universal availability and excellent mechanical properties. Lee et al. concluded that the Young's modulus of cellulose nanofibers is between 100 and $160 \mathrm{GPa}$ (based on the work of various research groups) [7], which is impressive and appealing. One of the major challenges that nanocelluloses have to face is achieving the proper dispersion in a hydrophobic polymer matrix, which is mandatory for mechanically enhanced plastics. There are a few methods that 
have been known to improve the dispersion of NCs in polymeric matrix, such as grafting and other types of surface modification [8].

One potential way to improve the mechanical properties of biocomposites that is rather rarely used is to use a hybrid matrix consisting of interpenetrating or semi-interpenetrating polymer networks (IPNs and SIPNs). IPNs and SIPNs were one of the key classes of polymeric materials in the late 1960s and 1970s, and a major part of the research was conducted in that era. Lately, IPN and SIPN hybrid materials have been used especially for biomedical applications [9-11]. By definition, IPNs comprise an interwoven matrix of two chemically distinct polymers that are intimately mixed with each other with nominal bonding and that are both present as a continuous phase. In IPNs, an inter-tangled network is present among the polymers, which can be achieved by the inhibition of phase segregation by a crosslinked network structure [12]. This allows superimposition of the properties of both (or many) polymers. The primary difference between IPN and SIPN is that in IPN, both of the participating polymers are crosslinked, while in SIPNs, only one of the polymer crosslinked while other is kept in thermoplastic phase [13]. IPNs and SIPNs provide an interesting underutilized approach for preparing biocomposites, with improved performance. Aoki et al., in their work on cellulose acetate/poly (meth methacrylate), demonstrated the relevance of cellulose-based materials in "design of novel polymer composites" [14].

For the purpose of creating SIPNs, polylactic acid (PLA) and polyurethane (PU) were used in this study; both are well-known commercial polymers. PLA has a distinct advantage in that it can be produced from natural resources such as corn starch. In fact, it is one of the widely researched polymer with cellulose-based reinforcements [15-17]. Additionally, it has excellent Young's modulus and tensile strength. However, it is very brittle and has low impact resistance and toughness. The remedy for brittleness has been reported to be plasticization [18], blending [19], and rubber (elastomer) toughening [20]. In this work, we have used elastomeric PU to form a crosslinked co-continuous network, which is expected to improve the toughness without a significant decrease in the tensile properties. Additionally, using cellulose nanocrystals as reinforcements can be a disguised advantage to interpenetrating networks; having rod shaped morphology, they are capable of making their own network above percolation threshold [21- 
23]. Marcovich et al. the confirmed the percolation threshold of cellulose nanocrystals suspension by rheological measurement and found that it is below $1 \mathrm{wt} \%$, further they also reported strong interaction between the cellulose nanocrystals and the PU-matrix due to chemical reaction between $\mathrm{OH}$ groups on cellulose and the isocyanate component [23]. Therefore, we suggest that cellulose nanocrystals can create their own interpenetrating network within the PU network.

In the current work, our aim is to investigate the formation of interpenetrating networks for biobased nanocomposites. We also report the properties of an SIPN nanocomposite based on PLA, PU and cellulose nanocrystals. Special emphasis is given to the study on phase behavior by diverse instrumental and analytical techniques.

\section{Experimental}

\subsection{Materials}

PLA, with a melt flow index of $8 \mathrm{~g} / 10 \mathrm{~min}$, was supplied in pellet form by FUTERRO (Escanaffles, Belgium). The castor oil-based polyol (CO-polyol), Jagropol-400, was supplied by Jayant Agro-Organics, Mumbai. It has a hydroxyl value of $350 \mathrm{mg} \mathrm{KOH} / \mathrm{gm}$, a viscosity of 700-1100 mPa.s, and an average functionality of 3. Freeze-dried cellulose nanocrystals (2012FPL-CNC-043) from cellulose pulp were supplied by the USDA Forest Products Laboratory, Madison, USA. The specific charge on the surface of the CNCs was $19.005 \mathrm{C} / \mathrm{g}$ (determined by polyelectrolyte titration). The 4,4'-diphenylmethane diisocyanate (MDI), Lupranate ${ }^{\circledR}$ M20S, was supplied by BASF SE, (Ludwigshafen, Germany). The isocyanate content is reported to be approximately $32 \%$ with a viscosity of $250 \mathrm{mPa}$.s. The 1,4-dioxane (henceforth referred to as dioxane), with 99.5\% purity, was purchased from VWR, Sweden (manufactured by Merck KGaA). Distilled water was used wherever needed. The PLA and CO-polyol were vacuum dried at $70{ }^{\circ} \mathrm{C}$ for 4 hours and 24 hours, respectively, to remove any trace of moisture.

\subsection{Dispersion of $\mathrm{CNC}$ in $\mathrm{CO}$-polyol}

A stable dispersion of $3 \mathrm{wt} \% \mathrm{CNC}$ in water was initially prepared by stirring overnight on a magnetic stirrer. Then $100 \mathrm{~g}$ of $3 \mathrm{wt} \% \mathrm{CNC}$ suspension and $10 \mathrm{~g}$ of CO-polyol were taken in a 
glass beaker and vigorously stirred at $12000 \mathrm{rpm}$ for 5 minutes with an Ultra-Turrax homogenizer. The charged CNCs act as an in-situ surfactant, leading to a milky white oil in water suspension. The dispersion was kept on the magnetic stirrer equipped with a heating plate, and a star-shaped magnet was used to generate high shear and the rpm was adjusted until a vortex was formed. The water in the dispersion was gradually removed by heating the dispersion at $75{ }^{\circ} \mathrm{C}$ and was slowly replaced with CO-polyol with the help of dioxane. Over a period of three hours, $100 \mathrm{~g}$ of dioxane and $50 \mathrm{~g}$ of CO-polyol were gradually added. The mixture was heated for three additional hours to remove volatiles and was kept in a vacuum overnight at $70{ }^{\circ} \mathrm{C}$ to remove traces of water and dioxane. Optical microscopy was used to monitor the quality of dispersion.

\subsection{Preparation of SIPNs and SIPN nanocomposites}

SIPN and SIPN nanocomposites, with PLA as the major phase, were prepared by solution casting. Initially, PLA $(5 \mathrm{wt} \%)$ was dissolved by stirring the pellets in dioxane for around 12 hours at room temperature. A calculated amount of CO-polyol was added to a part of the PLA solution according to required formulations, which are presented in Table 1. Isocyanate was subsequently added to the PLA-CO polyol solution while stirring. The PLA-CO polyolisocyanate solution (which was transparent) was cast in a Teflon petri dish and dried under vacuum for 12 hours at room temperature. In order to complete crosslinking and remove warpage due to thermal stresses, the film was compression molded at $175^{\circ} \mathrm{C}$ under a pressure of 1-2 MPa. The residual solvent was removed by annealing the films in a vacuum oven at $75^{\circ}$ $+/-5^{\circ} \mathrm{C}$ for 24 hours. Neat PU films were cast by mixing the isocyanate and CO-polyol and thinning down to around $50 \mathrm{wt} \%$ solid content. The mixture was stirred on a magnetic stirrer and cast on a Teflon mold. All the curing conditions for PU were kept the same except for hot pressings, as neat thermosets cannot be melt pressed again.

For the nanocomposite, CNC-dispersed CO-polyol was used instead of pure CO-polyol, while the rest of process remained the same. Additionally, the mixture was sonicated for 5 minutes before casting to facilitate $\mathrm{CNC}$ dispersion. The thickness of the films was around $150 \mu \mathrm{m}$ for PLA and SIPNs. On the other hand, PU films were around $2.5 \mathrm{~mm}$ thick due to the inability to cast a thin film from a low-viscosity thermoset on a Teflon mold, which has high surface energy. 
Table 1. Formulations of neat PLA, neat PU, SIPN, and SIPN nanocomposite films (on a nonvolatile materials basis).

\begin{tabular}{lcccc}
\hline \multicolumn{5}{c}{ Sample name } \\
\hline Materials & Neat PLA & SIPN & SIPN1CNC & \\
PLA & film & Film & film & Neat PU film \\
CO-Polyol & 100 & 70 & 69.3 & 0 \\
CO-Polyol 5 CNC & 0 & 18 & 0 & 60.1 \\
Isocyanate & 0 & 0 & 18.8 & 0 \\
\hline Total & 0 & 12 & 11.9 & 39.9 \\
\hline
\end{tabular}

\subsection{Characterization}

The gel content of the SIPN and SIPN-nanocomposite samples was analyzed by solvent extraction. Samples were cut in the form of rectangles and stirred in dioxane solvent on the magnetic stirrer. The samples were removed after 2 hours and were dried overnight in order to remove the dioxane. Accelerated vacuum drying was used for the PU samples due to their thickness. The initial and final weights were used to calculate the gel content according to the following formula:

Where and represent the weight of samples before and after extraction, respectively.

The optical images were gathered by a Nikon Eclipse LV100N POL optical microscope with software NIS-Elements D 4.30.02. The images were used to characterize the dispersion state and efficiency of the dispersion method.

The size of the CNCs was characterized by an atomic force microscope (AFM) with a multimode scanning probe (Veeco, Santa Barbara, USA) from TESPA tips (Bruker, Camarillo, USA). AFM height images were generated by the tapping mode in air.

The morphology of the SIPNs and the nanocomposite was characterized by field-emission scanning electron microscopy (FESEM) Zeiss Ultra Plus (Oberkochen, Germany). The samples were fractured in liquid nitrogen for imaging. All the samples were mounted on clips vertically and were coated with platinum to avoid charging, and an acceleration voltage of $5 \mathrm{kV}$ was used for imaging. The secondary electron mode was used to collect signals from the surface. 
The mechanical properties of the samples were evaluated by tensile tests, which were conducted with a Shimadzu AG-X universal testing machine (Kyoto, Japan), with $1 \mathrm{kN}$ load cell, a gauge length of $20 \mathrm{~mm}$, and a crosshead speed of $3 \mathrm{~mm} / \mathrm{min}$. Samples were cut into rectangular strips of $70 \times 5 \mathrm{~mm}$. Before testing, the samples were conditioned for at least 48 hours at room temperature $\left(23 \pm 2{ }^{\circ} \mathrm{C}\right)$ and $55 \pm 5 \%$ relative humidity. The results are reported as an average of a minimum of 7 samples. The elastic modulus was determined in the linear region of the stressstrain curve ( $1 \%$ to $3 \%$ elongation), and toughness was calculated from the area under the curve.

The dynamic mechanical properties of the samples were determined by DMA Q800 TA Instruments (New Castle, USA). Samples were cut into rectangular strips (40 x $5 \mathrm{~mm}$ ), and the tests were conducted in tension mode at an amplitude of $10 \mu \mathrm{m}$ and a frequency of $1 \mathrm{~Hz}$. The heating rate was kept constant to $3{ }^{\circ} \mathrm{C} / \mathrm{min}$, and the values of the storage modulus, loss modulus, and tan delta were recorded in the range of $-5^{\circ} \mathrm{C}$ to $120^{\circ} \mathrm{C}$. Liquid nitrogen was used as the coolant.

The differential scanning calorimetry (DSC) of samples was conducted with Mettler Toledo DSC 822e equipment (Schwerzenbach, Switzerland). In an aluminum pan, 6-10 mg of sample was taken, and graphs were recorded with the empty pan as a reference. The samples were first cooled to $-70{ }^{\circ} \mathrm{C}$ and were heated to $200{ }^{\circ} \mathrm{C}$ and cooled again to $-70{ }^{\circ} \mathrm{C}$ (in order to remove the thermal history). Finally, a subsequent scan was conducted again to $200{ }^{\circ} \mathrm{C}$, which was used for the analysis of the thermal characteristics. The heating rate for all the cycles was $10{ }^{\circ} \mathrm{C} / \mathrm{min}$. STARe SW 13.00 software was used to evaluate the data. The midpoint of the heat capacity change is reported as the glass transition temperature $\left(T_{g}\right)$. For the calculation of crystallinity, $93 \mathrm{~J} / \mathrm{g}$ was used as the enthalpy of fusion of neat PLA, as reported by Fischer et al. [24]. Thermogravimetric analysis of the samples was conducted with a Netzsch STA 449 F3 TGDTA/DSC analyzer (Selb, Germany). Samples of weight around $10 \mathrm{mg}$ were kept in a tungsten furnace with a heating rate of $10{ }^{\circ} \mathrm{C} / \mathrm{min}$ up to $700{ }^{\circ} \mathrm{C}$, under inert conditions. The TGA and DTG curves were recorded for each sample. 


\section{Results and Discussion}

\subsection{Determination of matrix composition}

To ensure the eco-friendly nature of the material, it was decided that PLA should be kept as a major component of the SIPN. The final concentration of PLA and PU in the matrix was based on the results of a preliminary experimental study in which concentrations of the PLA, COpolyol, and isocyanate were independent variables, and tensile properties were response variables. The results are presented in Table 2 . The coding of samples in preliminary studies for determination of matrix composition are as follows, the samples are coded in the form of SIPN $\mathrm{X}(100-\mathrm{X})$ is the sample of SIPNs where $\mathrm{X}$ and $(100-\mathrm{X})$ are the weight percentages of PLA and PU, respectively. For example, SIPN 8020 represents a sample that has SIPN formed with 80 $\mathrm{wt} \%$ PLA and $20 \mathrm{wt} \%$ PU. The number in brackets in the sample code represents the ratio of the isocyanate and hydroxyl $(\mathrm{NCO} / \mathrm{OH})$ in the polyurethanes.

In brief, the SIPN samples were prepared with a PLA:PU of 80:20 and 70:30, and the $\mathrm{NCO} / \mathrm{OH}$ ratio was kept at two levels, 0.8 and 0.6 , in both samples. Based on the results presented in Table 2, it can be observed that the SIPN with the PLA:PU ratio of 70:30 and the NCO/OH at the 0.8 level displayed optimized tensile properties. The same SIPN combination was used to prepare a nanocomposite reinforced with CNC. For the purpose of brevity, this particular combination of PLA and PU is referred simply as SIPN in the future text.

Table 2 Quantitative results from stress-strain analysis of neat PLA and SIPNs (along with their composition). The results indicate the average values of elastic modulus, elongation, tensile strength, and toughness (area under stress strain curve).

\begin{tabular}{ccccc}
\hline Sample name & $\begin{array}{c}\text { E-modulus } \\
(\mathbf{M P a})\end{array}$ & $\begin{array}{c}\text { Elongation } \\
\mathbf{( \% )}\end{array}$ & $\begin{array}{c}\text { Strength } \\
\mathbf{( M P a})\end{array}$ & $\begin{array}{c}\text { Toughness } \\
\left.\mathbf{( M J} / \mathbf{m}^{3}\right)\end{array}$ \\
\hline Neat PLA & $1699 \pm 50^{\mathrm{a}}$ & $4.2 \pm 0.4^{\mathrm{a}}$ & $59 \pm 2^{\mathrm{a}}$ & $152 \pm 23^{\mathrm{a}}$ \\
SIPN 7030 (0.6) & $544 \pm 48^{\mathrm{b}}$ & $9.8 \pm 2.0^{\mathrm{b}}$ & $24 \pm 1.5^{\mathrm{b}}$ & $189 \pm 50^{\mathrm{b}}$ \\
SIPN 8020(0.6) & $816 \pm 64^{\mathrm{c}}$ & $4.5 \pm 1.0^{\mathrm{c}}$ & $30 \pm 2^{\mathrm{c}}$ & $91 \pm 31^{\mathrm{c}}$ \\
SIPN 7030(0.8) & $911 \pm 103^{\mathrm{d}}$ & $13.6 \pm 3.8^{\mathrm{d}}$ & $38 \pm 4.5^{\mathrm{d}}$ & $437 \pm 162^{\mathrm{d}}$ \\
SIPN 8020(0.8) & $1035 \pm 98^{\mathrm{e}}$ & $7.4 \pm 1.7^{\mathrm{e}}$ & $40 \pm 2^{\mathrm{e}}$ & $219 \pm 57^{\mathrm{e}}$ \\
\hline
\end{tabular}

*Means that are marked by different superscript letters within the same column are significantly different at $5 \%$ level based on the analysis of variance (ANOVA). 


\subsection{Gel content}

Solvent extraction is commonly used to characterize the properties of SIPNs [25] and IPNs [26] by removing the thermoplastic and the non-crosslinked part of the polymer from the materials. In fact, it provides an easy confirmation that polymers indeed form an interpenetrating network and not a blend. A continuous porous network after dissolution of a non-crosslinked polymer indicates a co-continuous network. The true morphology can also be understood better after the dissolution of one phase. Table 3 presents the results from the solvent extraction of the SIPNs. It can be observed that the gel content is proportional to or slightly higher than the amount of thermoset (PU) in SIPN. The gel content is increased either by crosslinking or by an increase in molecular weight [27]. Based on the result from the neat PU sample for the amount of gel content in the 7030 SIPN, the estimated gel content should be $27 \%$; however, actual results indicated a higher gel content (34\%). This higher gel content can be explained based on the chemical structure of PLA, which has a terminal hydroxyl group capable of crosslinking with the isocyanate group and thus contributing to the crosslinked mass. This is likely to add the advantage of interfacial stabilization between two phases. Harada et al. have also reported that isocyanate can be used as a reactive processing agent in a PLA and poly(butylene succinate) blend to improve interfacial adhesion between polymer phases [28].

Table 3. Gel content of the prepared SIPNs with neat PLA and neat PU (as references). The $\mathrm{NCO} / \mathrm{OH}$ ratio was kept at 0.8 in the SIPNs and the neat PU.

\begin{tabular}{ccc}
\hline Sample name & Sample size & Gel content $\left(\mathrm{W}_{\mathrm{f}} / \mathrm{W}_{\mathrm{i}} * 100\right)$ \\
\hline Neat PLA & 0.042 grams & $0 \%$ \\
SIPN $8020(0.8)$ & 0.052 grams & $23 \%$ \\
SIPN 7030 (0.8) & 0.082 grams & $34 \%$ \\
Neat PU & 0.645 grams & $91 \%$ \\
\hline
\end{tabular}

Figure 1 (a) represents the photographic image of the SIPN film before and after PLA extraction. It is worth mentioning that the solutions before film casting were of a brownish transparent liquid, while the SIPN films were translucent in nature. The translucent nature of the films indicates phase separation, which is one necessary condition for the formation of SIPNs. It is well known fact that phase separation can result in turbidity if the phase domain size is larger 
than the wavelength of visible light [29]. Figure 1 (b) represents the optical microscopy images of the SIPN film, showing the PU network that remained after the extraction of the PLA. Fig 1 (b) confirms that the PU formed a network among PLA; the white areas represent the places where PLA was in the original films.

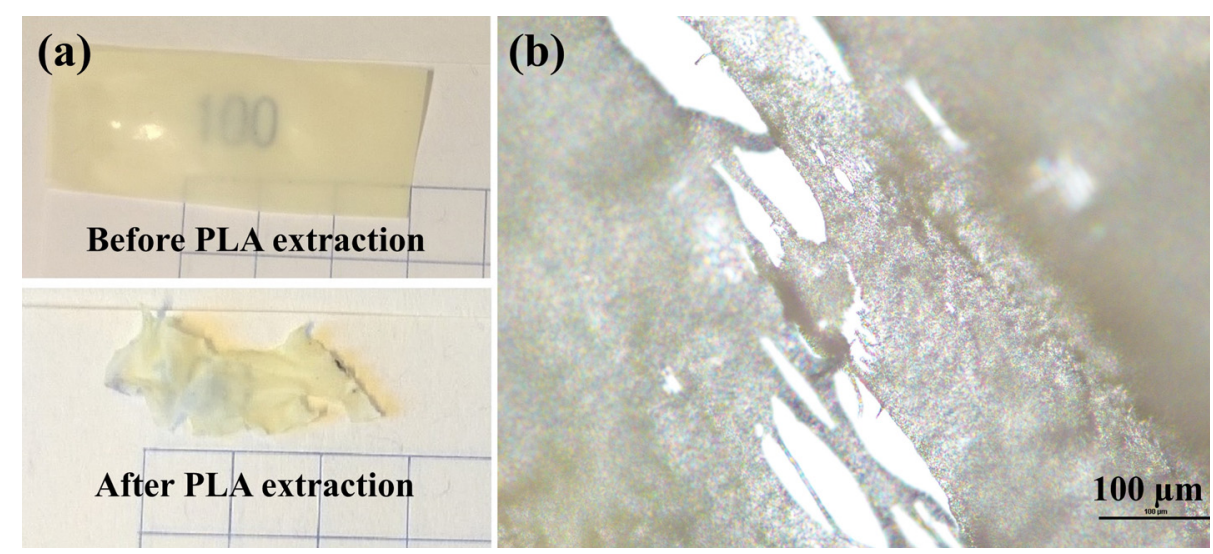

Figure 1. Visual appearance and microstructure of the SIPN: (a) SIPN before and after solvent extraction of PLA; continuous film after represents PU network; (b) optical microscopy image of SIPN after extraction of PLA; white pores represent the area where PLA was present in the original films.

\subsection{Dispersion of $C N C$}

Sonication is reported as one of the most popular methods for dispersing nanoparticles in a liquid medium [30]. Therefore, our first attempt used sonication to disperse CNCs in the COpolyol, which is a rather viscous liquid. After 2 hours sonication of dried CNCs in the polyol, we found that the dried CNC flakes were not dispersible in the polyol, as seen in the optical microscopy image in Figure 2 (b). The true size of CNCs is in nanoscale order shown in Figure 2 (a), which represents the AFM image of dried CNCs (from a water dispersion). The visible agglomerates in Figure 2 (b) show that the nanocrystals were not individually separated and hence, were not well dispersed.

CNCs, used in this study, are able to form a stable suspension in water. A novel method was designed in order to achieve fine dispersion by using a co-solvent to transfer dispersion from the water phase to the organic phase (the polyol phase). The method, henceforth called the cosolvent-assisted method, was designed considering the phase diagram for three components that are partially miscible with each other (ternary phase diagrams) [31]. Dioxane, which is compatible with water and CO-polyol, was used as the co-solvent. The compatibility 
(homogeneity) of water, CO-polyol, and dioxane was increased by high shear forces and temperature [32]. The CNCs were stabilized in water and the polyol mixture, by dioxane and volatiles were gradually removed from the mixtures, leaving $\mathrm{CNC}$ dispersed in $\mathrm{CO}$-polyol. The dioxane acted as a bridge between the water and the CO-polyol, balancing the solubility parameter and preventing the system from collapsing (separation) during the transition.

The co-solvent-assisted method demonstrated in this work produced satisfactory results, which are presented in Figure 2 (c). It can be seen that excellent dispersion was achieved when compared to the results from sonication. It is worth mentioning that this method produced successful results up to $6 \mathrm{wt} \% \mathrm{CNC}$ in the CO-polyol. Above that, a cake-like separation was observed over the dried polyol. The credit for the success of this method should be given to the chemical structure of castor oil, which has a hydroxyl group pendant to the long polymer chain, which is likely to have some stability in tandem with a hydroxyl group of CNC. The effectiveness of this method is further strengthened by the enhanced mechanical properties discussed in mechanical properties section.

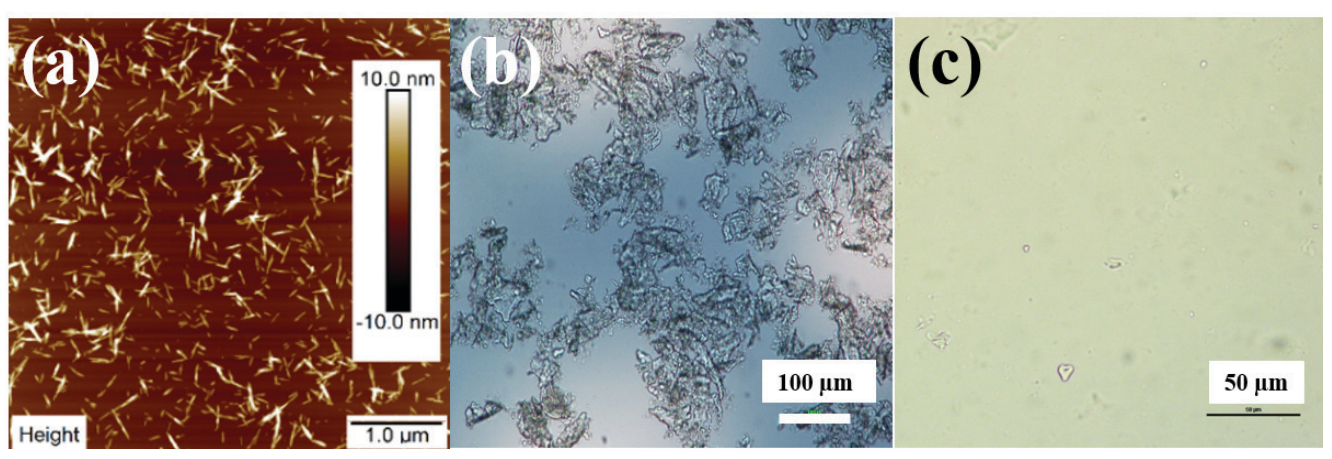

Figure 2. Optical microscopy images from dispersion studies of CNC in the CO-polyol: (a) AFM image of dried CNCs indicating the true size of individual CNCs, which is nanoscale: (b) $\mathrm{CNC}$ in CO-polyol after 2 hours of intense sonication; agglomerates are easily seen of tens of microns; (c) CNC in CO-polyol dispersed by co-solvent-assisted method; no agglomerates are visible, indicating a well dispersed CNC.

\subsection{Microstructure}

Figure 3 represents a fractured cross-section of SIPN reinforced with CNC. This image reveals the continuous threadlike appearances, dispersed in the PLA matrix, which refer to the PU network. The fibrillar PU network confirms the co-continuous network, which is characteristic of interpenetrating networks. 


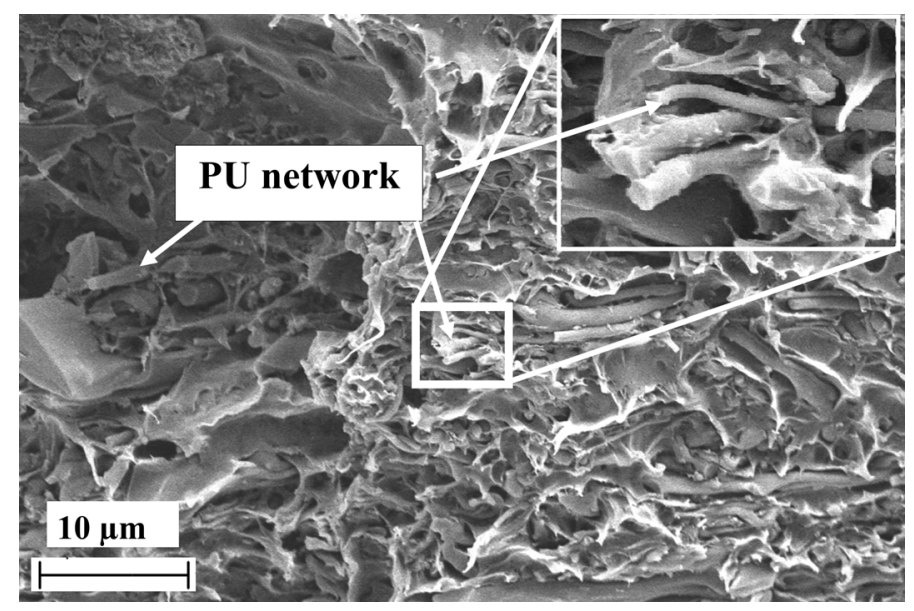

Figure 3. Microstructure of fractured SIPN nanocomposite; PU network is visible, confirming co-continuous morphology; fibrillar appearance of PU network is also visible.

\subsection{Mechanical properties}

The success of the co-solvent-assisted method was confirmed by mechanical testing. A comparison of tensile properties was done between PU and the PU nanocomposite reinforced with CNC. The PU nanocomposite with $0.7 \mathrm{wt} \% \mathrm{CNC}$ was prepared for tensile testing with CNC-dispersed CO-polyol. The $\mathrm{NCO} / \mathrm{OH}$ ratios in the neat $\mathrm{PU}$ samples were kept at 0.8 and 0.6. The stress-strain curves are presented in Figure 4. Homogeneous dispersion of the CNC in the CO-polyol enhances the tensile properties of the CNC-reinforced PU over the neat PU. The tensile properties were significantly improved with the addition of even $0.7 \mathrm{wt} \% \mathrm{CNC}$, and this confirms the good dispersion assured from the optical microscopy.

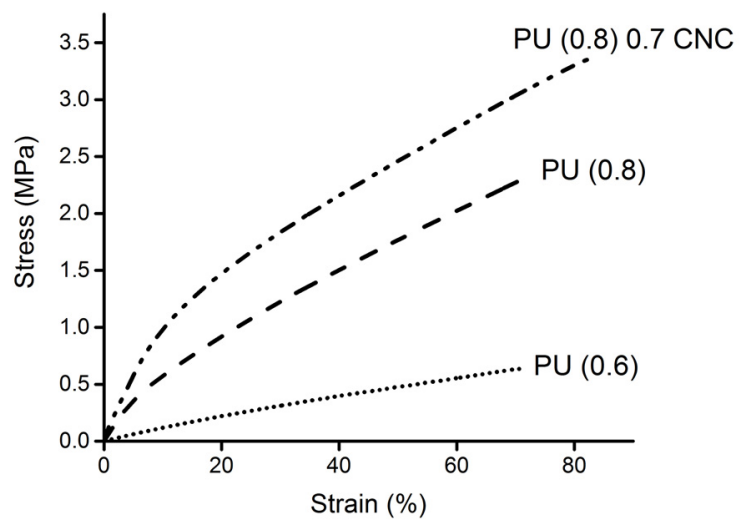

Figure 4. Stress-strain curves of $\mathrm{PU}$, with $\mathrm{NCO} / \mathrm{OH}$ ratio of 0.6 and 0.8 , and nanocomposite with $0.7 \mathrm{wt} \% \mathrm{CNC}$. The nanocomposite shows significant increased tensile properties, indicating excellent dispersion. 
Figure 5 presents the stress-strain curves of the neat PLA, neat PU, SIPN, and SIPN nanocomposite with $1 \mathrm{wt} \% \mathrm{CNC}$; the quantitative results are presented in Table 4 . The samples are coded in following way: Neat PLA and PU are represented as the same, SIPN with 70 wt. \% PLA and 30 wt. \% PU (with NCO/OH ratio 0.8) is represented as SIPN, and same SIPN reinforced with $1 \mathrm{wt} \% \mathrm{CNC}$ is represented as SIPN1CNC. One-way analysis of variance (ANOVA) indicated significant statistical differences $(p<0.05)$ in the tensile properties of the materials tested.

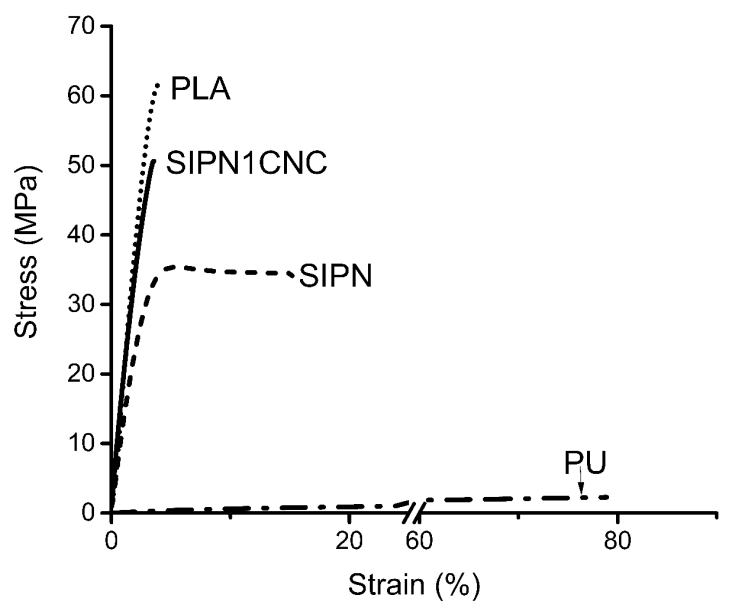

Figure 5. Stress-strain curves of PLA, PU, SIPN, and SIPN nanocomposite, indicating a superimposed behavior of SIPN and the reinforcing potential of CNCs even at as low a concentration as $1 \mathrm{wt} \%$.

The elongation of the used PU is high (around 80\%), and the tensile modulus is extremely low (7.5 MPa) when compared to those of the neat PLA (4.2\% and $1.7 \mathrm{GPa}$, respectively). If comparing the neat materials to the SIPN and the SIPN nanocomposites, the elongation of the prepared SIPN 7030 increased by $14 \%$ as well as the toughness (Table 4), while the modulus and strength were decreased. However, these results are encouraging when viewed in the light of the information that the concentration of PU in SIPN is as high as $30 \mathrm{wt} \%$ and itself has very a low modulus. We were able to retain $56 \%$ of the stiffness and yet improve the toughness by $190 \%$. Many factors can be attributed to this behavior. Firstly, the PLA has terminal hydroxyl groups that are likely to react with the isocyanate and contribute to increased chain length and an increase in molecular weight. Additionally, the co-continuous morphology seen in Figure 3 is known to show self-reinforcement behavior, which contributes to strength [33]. Finally, castor oil-based PU and PLA are partially miscible, which is likely to result in strong interface 
adhesion between the phases [34]. The results indicate the success in an area of our primary interest, an improvement in the toughness of SIPNs with respect to PLA.

Table 4. Quantitative results from stress-strain analysis indicating the average values (along with standard deviation) of elastic modulus, elongation to break, tensile strength, and toughness (area under stress strain curve).

\begin{tabular}{ccccc}
\hline Materials & $\begin{array}{c}\text { E-modulus* } \\
(\mathrm{MPa})\end{array}$ & $\begin{array}{c}\text { Elongation to } \\
\text { break* }^{*} \%\end{array}$ & $\begin{array}{c}\text { Strength* } \\
(\mathrm{MPa})\end{array}$ & $\begin{array}{c}\text { Toughness* } \\
\left(\mathrm{MJ} / \mathrm{m}^{3}\right)\end{array}$ \\
\hline PLA & $1699 \pm 50^{\mathrm{a}}$ & $4.2 \pm 0.4^{\mathrm{a}}$ & $59 \pm 2^{\mathrm{a}}$ & $152 \pm 23^{\mathrm{a}}$ \\
SIPN & $911 \pm 103^{\mathrm{b}}$ & $13.6 \pm 3.8^{\mathrm{b}}$ & $38 \pm 4^{\mathrm{b}}$ & $437 \pm 162^{\mathrm{b}}$ \\
SIPN1CNC & $1408 \pm 86^{\mathrm{c}}$ & $3.3 \pm 0.5^{\mathrm{c}}$ & $46 \pm 4^{\mathrm{c}}$ & $85 \pm 19^{\mathrm{c}}$ \\
PU & $7.5 \pm 0.6^{\mathrm{d}}$ & $83 \pm 11.7^{\mathrm{d}}$ & $2.6 \pm 0.3^{\mathrm{d}}$ & $125 \pm 32^{\mathrm{d}}$ \\
\hline
\end{tabular}

*Means that are marked by different superscript letters within the same column are significantly different at $5 \%$ level based on the ANOVA.

For the SIPN nanocomposite, it can be observed that even at as low a concentration as $1 \mathrm{wt} \%$ $\mathrm{CNC}$, there is a significant increase in tensile properties (over the SIPN), confirming the effectiveness of the co-solvent-assisted method for CNC dispersion. However, despite the improvement in elastic modulus and tensile strength, toughness is decreased. The primary reason behind this might be the large reinforcing effect of the CNCs. DMA analysis, discussed later in this study, indicated that CNCs prefer the PU phase rather than the PLA phase, which makes the elastomeric phase stiffer. Additionally, this loss in toughness can be explained by an increase in crosslinking due to the presence of an additional hydroxyl group from the CNCs. It is well-known that CNCs have a pendant hydroxyl group capable of chemical reactions, and in this case, the presence of a reactive moiety such as isocyanate could result in a robust interface, hindering the relative movement of the PU soft phases, which are mandatory in high elongation. Rueda et al. have reported that the isocyanate-modified $\mathrm{CNC}$ interacts with hard segments of PU and enhances stiffness and reduces ductility [35]. Finally, the reduced toughness can also be attributed to increase in crystallinity of PLA phase, as shown in Table 5. The results are a bit disappointing, as we lost the toughness that was gained by making SIPNs; nevertheless, they do provide an interesting insight into the reinforcement of SIPNs with reactive nanoreinforcements such as CNCs. 


\subsection{Thermal properties}

Figure 6 presents the DSC thermograms of the neat PLA, SIPN, and SIPN nanocomposite. The quantitative results are presented in Table 4 . The $\mathrm{T}_{\mathrm{g}}$ of PLA and PU is $50{ }^{\circ} \mathrm{C}$ and $5{ }^{\circ} \mathrm{C}$, respectively. For the SIPN, the $\mathrm{T}_{\mathrm{g}}$ was $52^{\circ} \mathrm{C}$, which is higher than any of the components in the mixture alone. The increase was approximately $3.5 \%$. The primary reason behind this may be the co-continuous self-reinforced morphology of SIPN, as shown earlier in Figure 3.

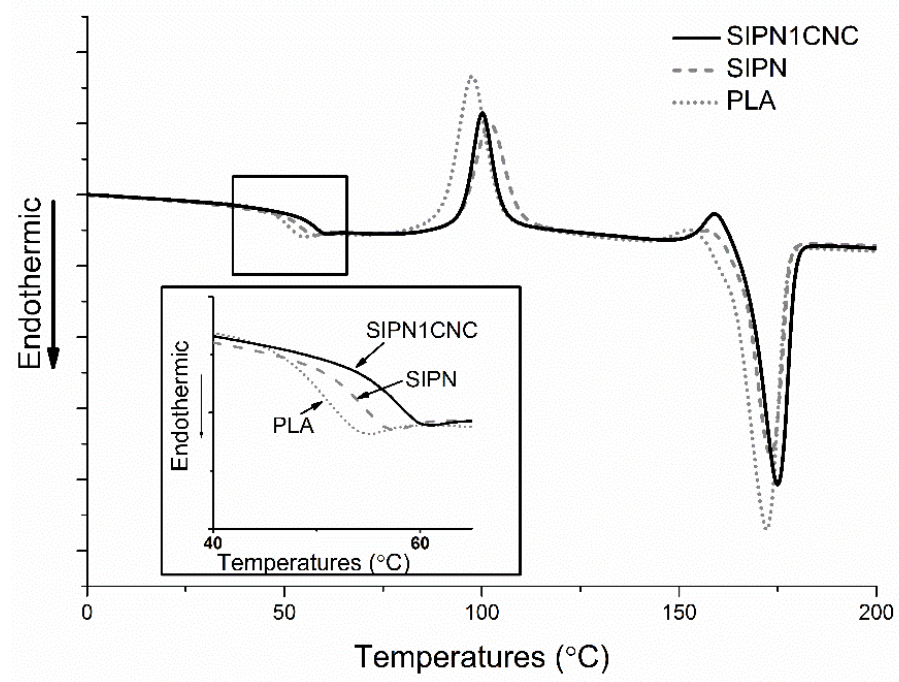

Figure 6. DSC thermograms for PLA, SIPN, and SIPN nanocomposite. The increase in $\mathrm{T}_{\mathrm{g}}$, represented by the inflexion of the thermograms from PLA to SIPN-nanocomposite through SIPN can easily be observed in the inset thermograms, which are the magnification of data points between $40{ }^{\circ} \mathrm{C}$ and $70{ }^{\circ} \mathrm{C}$. The melting and cold crystallization behavior can also be observed.

Additionally, the SIPN nanocomposite show a notable increase in $\mathrm{T}_{\mathrm{g}}$, being approximately $13 \%$ from $50{ }^{\circ} \mathrm{C}$ for PLA to $57^{\circ} \mathrm{C}$ for SIPN nanocomposite. The change in $\mathrm{T}_{\mathrm{g}}$ can be seen in the inset graph presented in Figure 6. On the other hand, the melt temperature $\left(\mathrm{T}_{\mathrm{m}}\right)$ also increases with the addition of $\mathrm{CNC}$; the increase, however, is nominal (around $2{ }^{\circ} \mathrm{C}$ ) at $1 \mathrm{wt} \% \mathrm{CNC}$. Additionally, the cold crystallization temperature is marginally increased in SIPN and in the SIPN nanocomposite over the neat PLA. The crystallinity of SIPN nanocomposite is also increased to $23 \%$ from $19 \%$ in neat PLA. A possible reason might be the crystallization of PLA phase in the presence of CNCs [36].

Table 5. Quantitative data from DSC analysis ( $\mathrm{T}_{\mathrm{g}}$ : glass transition temperature; Tcc: cold crystallization temperature; Tm: melting point and crystallinity) 


\begin{tabular}{lcccc}
\hline Sample name & $\begin{array}{c}\mathrm{T}_{\mathrm{g}} \\
\left({ }^{\circ} \mathrm{C}\right)\end{array}$ & $\mathrm{Tcc}\left({ }^{\circ} \mathrm{C}\right)$ & $\begin{array}{c}\mathrm{Tm} \\
\left({ }^{\circ} \mathrm{C}\right)\end{array}$ & $\begin{array}{c}\text { Crystallinity } \\
(\%)\end{array}$ \\
\hline PLA & 50 & 99 & 173 & 19.0 \\
SIPN & 52 & 100 & 173 & 13.5 \\
SIPN1CNC & 57 & 101 & 175 & 23.0 \\
PU & 5 & - & - & - \\
\hline
\end{tabular}

DMA study was made to study the neat polymers PU and PLA, the SIPN and the SIPN nanocomposite viscoelastic properties as a function of temperature. Figure 7(a) where the storage modulus $\left(\mathrm{E}^{\prime}\right)$ as function of temperature is seen, show that the PU is softening and breaking already around $60{ }^{\circ} \mathrm{C}$ and the neat PU and SIPN are visibly weaker than the neat PLA and SIPN nanocomposite. This was also expected from the tensile testing results. The most interesting observation is that the storage modulus of the SIPN nanocomposite is higher than that of the neat PLA polymer at temperatures higher than $55^{\circ} \mathrm{C}$. It remains higher up to $100{ }^{\circ} \mathrm{C}$. The reason might be the tandem effect of co-continuous morphology (which shows a slightly higher modulus in SIPNs above $85^{\circ} \mathrm{C}$ ) and the reinforcement efficiency of CNCs. Another possible explanation behind this can be that some CNC are trapped inside PLA phase, causing increase in modulus above $55^{\circ} \mathrm{C}$.

(a)

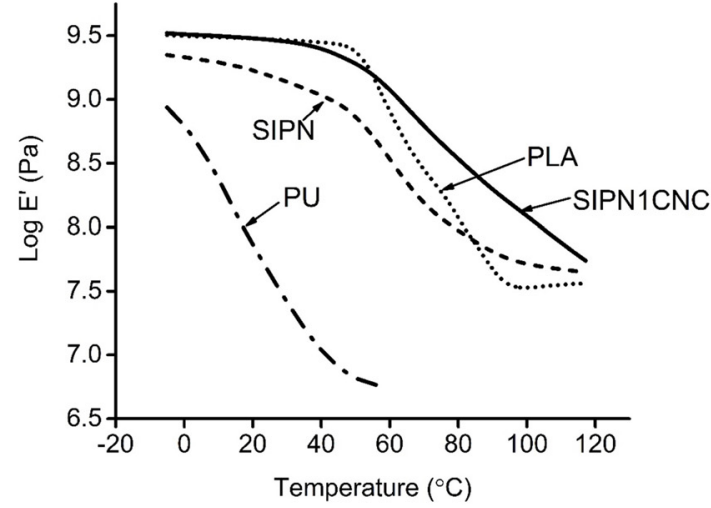

(b)

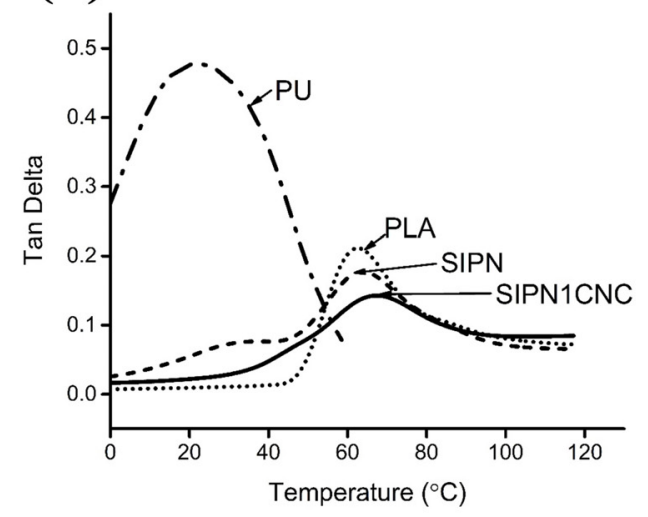

Figure 7. DMA thermograms of neat PLA, SIPN, SIPN nanocomposite, and neat PU, representing (a) storage modulus of samples with respect to temperatures. The modulus of the SIPN nanocomposite at temperatures below $25^{\circ} \mathrm{C}$ and above $60^{\circ} \mathrm{C}$ is higher than that of PLA, which is a surprising (b) tan delta at various temperatures. The most central one is a curve from the SIPN, which shows two separate peaks, one for the PU phase and one for the PLA phase. Interestingly, the PU curve is disappearing in the SIPN nanocomposite, indicating that $\mathrm{CNC}$ is reinforcing primarily that phase. 
The quantitative analysis of the reinforcement efficiency of $\mathrm{CNC}$ at various temperatures $(0,20$, 40 , and $80^{\circ} \mathrm{C}$ ) is presented in Table 6 . This increase in $\mathrm{E}^{\prime}$ is a result of a stiffening effect due to the presence of $\mathrm{CNC}$ and the interfacial interactions between polymer chains and CNCs. Additionally, the reinforcement efficiency (\% increase in $\left.\mathrm{E}^{\prime}\right)$ is higher above $\mathrm{T}_{\mathrm{g}}$, which is in agreement with earlier studies reinforcing efficiency of cellulose and other reinforcements is higher above $\operatorname{Tg}[37-40]$.

Table 6. Reinforcing efficiency of $\mathrm{CNC}$ at various temperatures $\left(0,20,40,80^{\circ} \mathrm{C}\right)$, indicating that reinforcement efficiency is highest at temperatures above $\mathrm{T}_{\mathrm{g}}$ of PLA.

\begin{tabular}{cccc}
\hline $\begin{array}{c}\text { Temperature } \\
\left({ }^{\circ} \mathrm{C}\right)\end{array}$ & $\begin{array}{c}\text { Storage modulus (SIPN) } \\
(\mathrm{MPa})\end{array}$ & $\begin{array}{c}\text { Storage modulus (SIPN1CNC) } \\
(\mathrm{MPa})\end{array}$ & $\begin{array}{c}\text { Increase } \\
(\%)\end{array}$ \\
\hline 0 & 2147 & 3243 & 51 \\
20 & 1690 & 3014 & 78 \\
40 & 1074 & 2498 & 133 \\
80 & 94 & 342 & 264 \\
\hline
\end{tabular}

Figure 7 (b) presents the tan delta curves for the neat PLA, neat PU, SIPN, and SIPN nanocomposite. The neat PU curve is around $23{ }^{\circ} \mathrm{C}$, and the neat PLA peak is around $63{ }^{\circ} \mathrm{C}$. SIPN has two distinct peaks at $34{ }^{\circ} \mathrm{C}$ (PU peak) and $64{ }^{\circ} \mathrm{C}$ (PLA peak). This twin peak is a significant characteristic of multi-phase polymeric materials when there is considerable phase separation [41], which in our case can be observed in the SEM image in Figure 3. The PU peak moves $11{ }^{\circ} \mathrm{C}$ closer to PLA peak (from $23^{\circ} \mathrm{C}$ to $34{ }^{\circ} \mathrm{C}$ ) in the SIPN, which can be explained by an increase in compatibility between the PLA and PU due to the isocyanate reaction with the terminal hydroxyl group of the PLA [34] as well as the crosslinking effect of the CNC.

There is another interesting observation in the tan delta curve of the SIPN nanocomposite, where the PU peak $\left(34{ }^{\circ} \mathrm{C}\right)$ is submerged in the PLA peak. Careful observation indicates that the PU peak moves to around $51{ }^{\circ} \mathrm{C}$. This can be explained by the presence of the CNCs, which preferably are reinforcing the PU phase; as a result, the tan delta peak of PU moves toward a higher temperature. The reason behind the CNC preference to PU phase can be explained by the chemical groups present during the crosslinking reactions. $\mathrm{CNC}$ has a pendant hydroxyl groups that are capable of reacting with isocyanate. Therefore, at a particular time, two types of crosslinking reactions are happening: $\mathrm{CO}$ polyol-isocyanate and $\mathrm{CNC}$-isocyanate. With chain 
propagation, CNCs become embedded in CO-PU chains and separate from the PLA. When the length (or molecular weight) of PU chains is large enough, PLA and PU phase separates from each other (as observed in Figure 3), with CNCs ending up in PU phase. A concentration of 1 wt $\%$ CNC in the PLA-PU SIPN, which primarily reinforces the PU phase, only results in $3.5 \mathrm{wt} \% \mathrm{CNC}$ in PU, and as Figure 4 indicates that just $0.7 \mathrm{wt} \% \mathrm{CNC}$ improves the stiffness of PU by a considerable amount, $3.5 \mathrm{wt} \%$ will definitely make it brittle. Therefore, the SIPN nanocomposite loses toughness. However, at this high concentration in the PU phase, CNCs are likely to form a stiff percolating network of their own within the PU network, effectively reinforcing it, especially at a high temperature, as observed in Figure 7 (a). Furthermore, the peak of PLA phase is also slightly shifting towards right. A possible explanation might be that some CNCs are trapped in PLA phase (due to terminal hydroxyl groups in PLA chains) and increasing the stiffness above $55{ }^{\circ} \mathrm{C}$. This interpretation is supported by the fact that crystallinity of nanocomposite is increasing (Table 5). CNCs are known to increase crystallinity of PLA films [36].

The TGA results of the PLA, PU, their SIPN, and the SIPN nanocomposite are presented in Figure 8. The advantage of making an interpenetrating network can be seen in Figure 8 (a); It can be seen from Figure 8 (a) that PU has higher extent of degradation (wt \%), and PLA is rather stable below $250^{\circ} \mathrm{C}$, but their combination is more stable than either of them alone. Additionally, PLA degrades rapidly above $350^{\circ} \mathrm{C}$, and PU is relatively more stable; as a result, their combination, SIPN, resists degradation above $350{ }^{\circ} \mathrm{C}$ (compared to the neat PLA). Among all the samples, the SIPN nanocomposite has the highest thermal stability. The benefit of the CNC can be clearly observed over SIPN and neat PLA (until $300^{\circ} \mathrm{C}$ ).

The DTG curves provide information relevant to the various steps of degradation in the polymers, if any. Figure 8 (b) presents the DTG curves of the samples; it can be observed that PLA has very rapid (one-step) degradation after $300{ }^{\circ} \mathrm{C}$, while $\mathrm{PU}$, on the other hand, has a three-step degradation and has a stronger heat tolerance, as a major part is degraded beyond $400{ }^{\circ} \mathrm{C}$. As a result, SIPN and the SIPN nanocomposite displayed stronger heat tolerance than the neat polymers. The quantitative analysis of the DTG results, consisting of the percent of decomposition between $0{ }^{\circ} \mathrm{C}$ and $400{ }^{\circ} \mathrm{C}$ and at temperatures corresponding to a $98 \%, 50 \%$, and 
$2 \%$ weight loss, is provided in Table 7 . The SIPNs and SIPN nanocomposite are respectively degraded $14 \mathrm{wt} \%$ and $16 \mathrm{wt} \%$ less than neat PLA between $0{ }^{\circ} \mathrm{C}$ and $400{ }^{\circ} \mathrm{C}$. Additionally, for temperature corresponding to a $2 \mathrm{wt} \%$ loss, the value for the nanocomposite is significantly higher $\left(270{ }^{\circ} \mathrm{C}\right)$ than for the PLA $\left(130^{\circ} \mathrm{C}\right)$ and the SIPN $\left(145^{\circ} \mathrm{C}\right)$, suggesting their improved heat tolerance. This is an additional benefit of making a nanocomposite from an SIPN. The degradation of castor oil-based PU has been reported by Hablot et al. [42] to be a three-step process, which is in agreement with our results. They mentioned that the first step (around 200 ${ }^{\circ} \mathrm{C}$ ) refers to the degradation of urethane groups, the second step (around $330{ }^{\circ} \mathrm{C}$ ) refers to the degradation of soft segments from the castor oil, and the third step (around $420{ }^{\circ} \mathrm{C}$ ) corresponds to the degradation of whatever is left over.

Table 7. Thermal analysis is indicating decomposition between $0{ }^{\circ} \mathrm{C}$ and $400{ }^{\circ} \mathrm{C}$ along with temperatures needed for $98 \%, 50 \%$ and $2 \%$ weight loss.

\begin{tabular}{lllll}
\hline Materials & $\begin{array}{l}\text { Decomposition } \\
\left(0-400{ }^{\circ} \mathrm{C}\right)(\%)\end{array}$ & $\mathrm{T}_{98 \%}\left({ }^{\circ} \mathrm{C}\right)$ & $\mathrm{T}_{50 \%}\left({ }^{\circ} \mathrm{C}\right)$ & $\mathrm{T}_{2 \%}\left({ }^{\circ} \mathrm{C}\right)$ \\
\hline PLA & 98.3 & 385 & 355 & 130 \\
SIPN & 84.3 & $>700$ & 325 & 145 \\
SIPN1CNC & 82.4 & $>700$ & 330 & 270 \\
PU & 56.6 & $>700$ & 375 & 110 \\
\hline
\end{tabular}

(a)

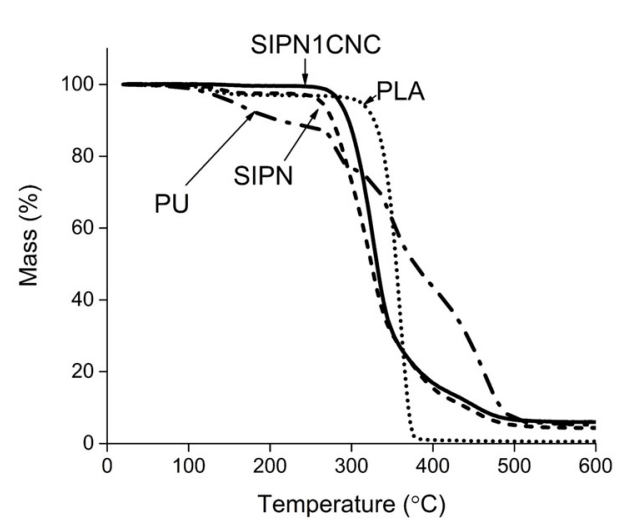

\section{(b)}

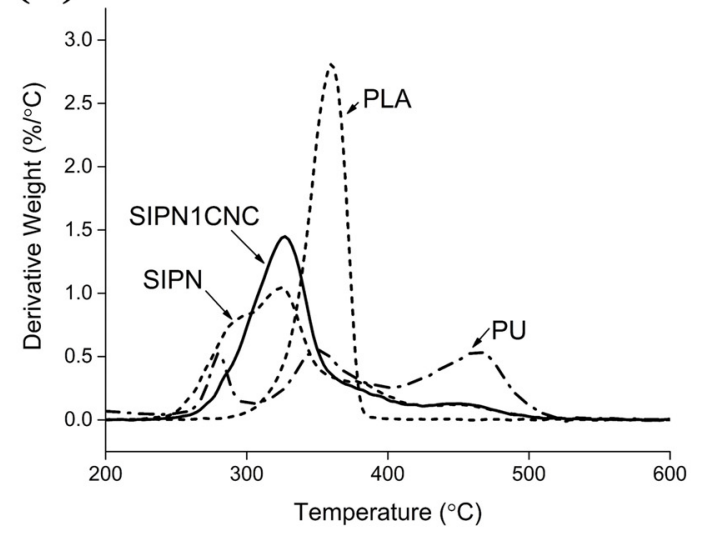

Figure 8. (a) TGA results indicate heat tolerance in the form of weight loss at various temperatures for neat PLA, neat PU, SIPN, and SIPN nanocomposite; (b) DTG results indicate the various stages of degradation for neat PLA, neat PU, SIPN, and SIPN nanocomposite. 


\section{Conclusion}

In this study, semi-interpenetrated networks (SIPNs) reinforced with cellulose nanocrystals derived from polylactic acid (PLA) and polyurethane (PU) polymers were prepared and characterized.

The co-solvent-assisted method used in this study provides a novel approach to disperse CNCs in a polyol phase without using surfactants.

Morphological analysis of the SIPN indicates that a thread-like PU network is formed in the PLA matrix with a strong interface.

The SIPN of the PLA-PU-reinforced CNCs provides multiple advantages: improved tensile properties, heat tolerance, and stiffness at higher temperatures. It is expected that the CNCs are also crosslinked with the isocyanate and form a stiff network in the PU phase. This network then hinders the movement of the PU chains very effectively and also results in a decrease in the toughness.

CNCs are also improving the crystallinity of PLA phase.

Further work can be conducted in order to improve the loss in ductility in the SIPN nanocomposites, possibly by decreasing the $\mathrm{CNC}$ content and/or using chain extenders.

\section{Acknowledgements}

The authors acknowledge the financial support of the TEKES FiDiPro Program. The authors would like to thank Dr. Nicole Stark at USDA Forest Products Laboratory FPL, USA, for kindly providing cellulose nanocrystals (2012-FPL-CNC-043). Finally, we would like to thank Robert Hartmann (University of Oulu, Finland) for his help with surface charge analysis of CNCs, Tommi Kokkonen (University of Oulu, Finland) with thermogravimetric analysis, and Shiyu Geng (Luleå University of Technology, Sweden) for atomic force microscopy.

\section{References}

[1] G. Lligadas, J.C. Ronda, M. Galià, V. Cádiz, Renewable polymeric materials from vegetable oils: A perspective, Mater. Today. 16 (2013) 337-343. doi:10.1016/j.mattod. 
2013.08.016.

[2] T.A. Hottle, M.M. Bilec, A.E. Landis, Sustainability assessments of bio-based polymers, Polym. Degrad. Stab. 98 (2013) 1898-1907. doi:10.1016/j.polymdegradstab. 2013.06.016.

[3] J.K. Pandey, A.N. Nakagaito, H. Takagi, Fabrication and applications of cellulose nanoparticle-based polymer composites, Polym. Eng. Sci. 53 (2013) 1-8. doi:10.1002/ pen.23242.

[4] S.A. Miller, A.E. Landis, T.L. Theis, Feature: Environmental Trade-offs of Biobased Production, Environ. Sci. Technol. 41 (2007) 5176-5182. doi:10.1021/es072581z.

[5] C. Johansson, J. Bras, I. Mondragon, P. Nechita, D. Plackett, P. Šimon, D.G. Svetec, S. Virtanen, M.G. Baschetti, C. Breen, F. Clegg, S. Aucejo, Renewable fibers and bio-based materials for packaging applications - A review of recent developments, BioResources. 7 (2012) 2506-2552. doi:10.15376/biores.7.2.2506-2552.

[6] L. Hojabri, X. Kong, S.S. Narine, Fatty acid-derived diisocyanate and biobased polyurethane produced from vegetable oil: Synthesis, polymerization, and characterization, Biomacromolecules. 10 (2009) 884-891. doi:10.1021/bm801411w.

[7] K.-Y. Lee, Y. Aitomäki, L.A. Berglund, K. Oksman, A. Bismarck, On the use of nanocellulose as reinforcement in polymer matrix composites, Compos. Sci. Technol. 105 (2014) 15-27. doi:10.1016/j.compscitech.2014.08.032.

[8] Y. Habibi, Key advances in the chemical modification of nanocelluloses., Chem. Soc. Rev. 43 (2014) 1519-42. doi:10.1039/c3cs60204d.

[9] Y. Zhang, Z. Fan, C. Xu, S. Fang, X. Liu, X. Li, Tough biohydrogels with interpenetrating network structure by bienzymatic crosslinking approach, Eur. Polym. J. 72 (2015) 717-725. doi:10.1016/j.eurpolymj.2014.12.038.

[10] S. Tanpichai, K. Oksman, Cross-linked nanocomposite hydrogels based on cellulose nanocrystals and PVA: Mechanical properties and creep recovery, Compos. Part A Appl. Sci. Manuf. 88 (2016) 226-233. doi:10.1016/j.compositesa.2016.06.002. 
[11] S. Butylina, S. Geng, K. Oksman, Properties of as-prepared and freeze-dried hydrogels made from poly(vinyl alcohol) and cellulose nanocrystals using freeze-thaw technique, Eur. Polym. J. 81 (2016) 386-396. doi:http://dx.doi.org/10.1016/j.eurpolymj. 2016.06.028.

[12] C.M. Roland, Interpenetrating polymer networks (IPN): Structure and mechanical behavior, Encycl. Polym. Nanomater. (2014) 1-9. doi:10.1007/978-3-642-36199-9.

[13] L.H. Sperling, Interpenetrating Polymer Networks and Related Materials, Springer US, 1981. doi:10.1007/978-1-4684-3830-7.

[14] D. Aoki, Y. Teramoto, Y. Nishio, Cellulose acetate/poly(methyl methacrylate) interpenetrating networks: Synthesis and estimation of thermal and mechanical properties, Cellulose. 18 (2011) 1441-1454. doi:10.1007/s10570-011-9580-5.

[15] A. Mujica-Garcia, S. Hooshmand, M. Skrifvars, J.M. Kenny, K. Oksman, L. Peponi, Poly(lactic acid) melt-spun fibers reinforced with functionalized cellulose nanocrystals, RSC Adv. 6 (2016) 9221-9231. doi:10.1039/C5RA22818B.

[16] M. Jonoobi, A.P. Mathew, M.M. Abdi, M.D. Makinejad, K. Oksman, A comparison of modified and unmodified cellulose nanofiber reinforced polylactic acid (PLA) prepared by twin screw extrusion, J. Polym. Environ. 20 (2012) 991-997. doi:10.1007/ s10924-012-0503-9.

[17] M. Jonoobi, J. Harun, A.P. Mathew, K. Oksman, Mechanical properties of cellulose nanofiber (CNF) reinforced polylactic acid (PLA) prepared by twin screw extrusion, Compos. Sci. Technol. 70 (2010) 1742-1747. doi:10.1016/j.compscitech.2010.07.005.

[18] S. Jacobsen, H.G. Fritz, Plasticizing polylactide - the effect of different plasticizers on the mechanical properties, Polym. Eng. Sci. 39 (1999) 1303-1310. doi:10.1002/pen. 11517.

[19] Y. Wang, M. Hillmyer, Polyethylene-poly (L-lactide) diblock copolymers: Synthesis and compatibilization of poly (L-lactide )/polyethylene blends, J. Polym. Sci. Part A Polym. Chem. 39 (2001) 2755-2766. doi:10.1002/pola.1254.

[20] C. Zhang, W. Wang, Y. Huang, Y. Pan, L. Jiang, Y. Dan, Y. Luo, Z. Peng, Thermal, 
mechanical and rheological properties of polylactide toughened by expoxidized natural rubber, Mater. Des. 45 (2013) 198-205. doi:10.1016/j.matdes.2012.09.024.

[21] M.A.S. Azizi Samir, F. Alloin, A. Dufresne, Review of Recent Research into Cellulosic Whisker, Their Properties and Their Application in Nanocomposites Field, Biomacromolecules. 6 (2005) 612-626. doi:10.1021/bm0493685.

[22] N.L. Garcia de Rodriguez, W. Thielemans, A. Dufresne, Sisal cellulose whiskers reinforced polyvinyl acetate nanocomposites, Cellulose. 13 (2006) 261-270. doi: 10.1007/s10570-005-9039-7.

[23] N.E. Marcovich, M.L. Auad, N.E. Bellesi, S.R. Nutt, M.I. Aranguren, Cellulose micro/ nanocrystals reinforced polyurethane, J. Mater. Res. 21 (2006) 870-881. doi:10.1557/ jmr.2006.0105.

[24] E.W. Fischer, H.J. Sterzel, G. Wegner, Investigation of the structure of solution grown crystals of lactide copolymers by means of chemical reactions, Kolloid-Zeitschrift Und Zeitschrift F $\{\ddot{u}\}$ r Polym. 251 (1973) 980-990.

[25] K. Zheng, J. Zhang, J. Cheng, Morphology, structure, miscibility, and properties of wholly soy-based semi-interpenetrating polymer networks from soy-oil-polyol-based polyurethane and modified soy protein isolate, Ind. Eng. Chem. Res. 52 (2013) 1433514341. doi:10.1021/ie401791v.

[26] N. Kayaman-Apohan, R. Demirci, M. Cakir, A. Gungor, UV-curable interpenetrating polymer networks based on acrylate/vinylether functionalized urethane oligomers, Radiat. Phys. Chem. 73 (2005) 254-262. doi:10.1016/j.radphyschem.2004.09.026.

[27] S.W. Lee, J.W. Park, C.H. Park, H.J. Kim, Y.G. Eom, UV-curing behaviors and mechanical properties of UV-cured polylactic acid (PLA), J. Korean Wood Sci. Technol. 41 (2013) 134-140. doi:10.5658/WOOD.2013.41.2.134.

[28] M. Harada, T. Ohya, K. Iida, H. Hayashi, K. Hirano, H. Fukuda, Increased impact strength of biodegradable poly(lactic acid)/poly(butylene succinate) blend composites by using isocyanate as a reactive processing agent, J. Appl. Polym. Sci. 106 (2007) 18131820. doi:10.1002/app.26717. 
[29] J. Yang, M.A. Winnik, D. Ylitalo, R.J. Devoe, Polyurethane-Polyacrylate Interpenetrating Networks. 1. Preparation and Morphology, Macromolecules. 9297 (1996) 7047-7054. doi:10.1021/ma9601373.

[30] P.C. Ma, N.A. Siddiqui, G. Marom, J.K. Kim, Dispersion and functionalization of carbon nanotubes for polymer-based nanocomposites: A review, Compos. Part A Appl. Sci. Manuf. 41 (2010) 1345-1367. doi:10.1016/j.compositesa.2010.07.003.

[31] M.L. Yeow, Y.T. Liu, K. Li, Isothermal phase diagrams and phase-inversion behavior of poly(vinylidene fluoride)/solvents/additives/water systems, J. Appl. Polym. Sci. 90 (2003) 2150-2155. doi:10.1002/app.12846.

[32] C. Krause, B.A. Wolf, R. Horst, B.A. Wolf, Shear effects on the phase diagrams of solutions of highly incompatible polymers in a common solvent . 2 . Experiment and theory, Macromolecules. 30 (1997) 890-895.

[33] Y.S. Lipatov, T. Alekseeva, Phase-Separated Interpenetrating Polymer Networks, Springer Berlin Heidelberg, Berlin, Heidelberg, 2007. doi:10.1007/978-3-540-73552-6.

[34] T. Gurunathan, S. Mohanty, S.K. Nayak, Preparation and performance evaluation of castor oil-based polyurethane prepolymer/polylactide blends, J. Mater. Sci. 49 (2014) 8016-8030. doi:10.1007/s10853-014-8509-4.

[35] L. Rueda, B. Fernández d'Arlas, Q. Zhou, L.A. Berglund, M.A. Corcuera, I. Mondragon, A. Eceiza, Isocyanate-rich cellulose nanocrystals and their selective insertion in elastomeric polyurethane, Compos. Sci. Technol. 71 (2011) 1953-1960. doi:10.1016/ j.compscitech.2011.09.014.

[36] E.M. Sullivan, R.J. Moon, K. Kalaitzidou, Processing and Characterization of Cellulose Nanocrystals/Polylactic Acid Nanocomposite Films, Materials. 8 (2015) 5447. doi: $10.3390 / \mathrm{ma} 8125447$.

[37] M.A. Saïd Azizi Samir, F. Alloin, M. Paillet, A. Dufresne, Tangling effect in fibrillated cellulose reinforced nanocomposites, Macromolecules. 37 (2004) 4313-4316. doi: $10.1021 / \mathrm{ma} 035939 \mathrm{u}$.

[38] A.P. Mathew, K. Oksman, M. Sain, The effect of morphology and chemical 
characteristics of cellulose reinforcements on the crystallinity of polylactic acid, J. Appl. Polym. Sci. 101 (2006) 300-310. doi:10.1002/app.23346.

[39] M.A. López-Manchado, M. Arroyo, Thermal and dynamic mechanical properties of polypropylene and short organic fiber composites, Polymer. 41 (2000) 7761-7767. doi: 10.1016/S0032-3861(00)00152-X.

[40] V. Hristov, S. Vasileva, Dynamic mechanical and thermal properties of modified poly(propylene) wood fiber composites, Macromol. Mater. Eng. 288 (2003) 798-806. doi:10.1002/mame.200300110.

[41] J.J. Fay, C.J. Murphy, D.A. Thomas, L.H. Sperling, Effect of morphology, crosslink density, and miscibility on interpenetrating polymer network damping effectiveness, Polym. Eng. Sci. 31 (1991) 1731-1741. doi:10.1002/pen.760312407.

[42] E. Hablot, D. Zheng, M. Bouquey, L. Avérous, Polyurethanes based on castor oil: Kinetics, chemical, mechanical and thermal properties, Macromol. Mater. Eng. 293 (2008) 922-929. doi:10.1002/mame.200800185. 